\title{
Association of CYP2C19, TNF- $\alpha$, NOD1, NOD2, and PPAR $\gamma$ polymorphisms with peptic ulcer disease enhanced by Helicobacter pylori infection
}

Laith N. AL-Eitan, MSc, PhD, Fouad A. Almomani, MSc, PhD, Sohaib M. Al-Khatib, MD.

\begin{abstract}

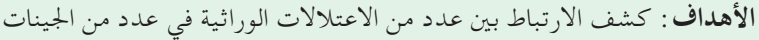

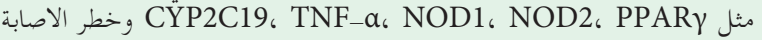
بجرثومة المعدة في المرضى الاردنيين.

المنهجية : تم استخراج وتحليل عينة الحمض النووي من الأنسجة المحفوظة بالبارافين

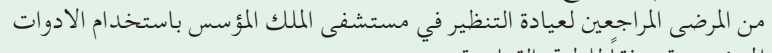

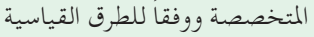

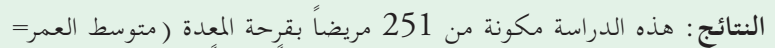

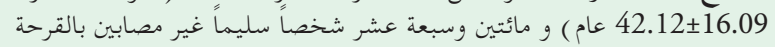

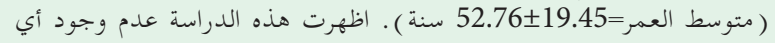

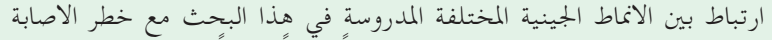

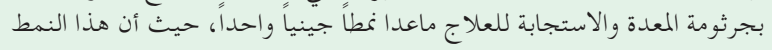

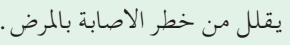

الحلاصة : قدمت هذة الدراسة على وجود ارتباط بين الاعتلالات الجاتلاتينية وخطر

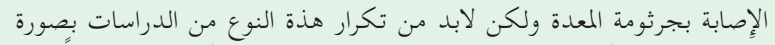

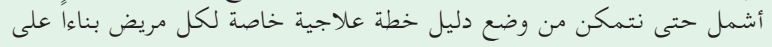
خارطتة الجينية
\end{abstract}

Objectives: To assess the correlation between a number of genetic variations of CYP2C19, TNF- $\alpha$, NOD1, NOD2, and PPAR $\gamma$ genes with the severity of Helicobacter pylori (H. pylori) infections and peptic ulcers (PU).

Methods: A retrospective cross-sectional design was used in this study. Formalin-fixed paraffin-embedded (FFPE) tissue was used to extract genomic DNA that was collected from Jordanian patients who visited endoscopy clinics between 2014 to 2018 at the King Abdullah University Hospital (KAUH), Irbid, Jordan. Genotyping of the studied single nucleotide polymorphisms (SNPs) were applied using the sequencing protocol.

Results: A total of 251 patients (mean age: $42.12 \pm 16.09$ years) and healthy controls (mean age: $52.76 \pm 19.45$ years) were enrolled in this study. This study showed no significant association between patients and the studied polymorphisms except for rs2075820 of the NOD1 $(p=0.0046)$. It is hypothesized that the heterozygous genotype (TC); $44.8 \%$ in patients versus $61.3 \%$ in controls has a decreased risk of peptic ulcers (OR: 0.49). The alleles frequency association was insignificant in all studied SNPs with a $p$-value more than 0.05 .

Conclusion: This study provided evidence regarding the association of the rs2075820 with $H$. pylori infections. The other studied SNPs were not statistically significant.

Keywords: Helicobacter pylori; peptic ulcers; cytochrome P-450; peroxisome proliferator-activated receptors; endoscopy

Saudi Med J 2021; Vol. 42 (1): 21-29 doi: 10.15537/smj.2021.1.25654

From the Department of Biotechnology and Genetic Engineering (AL-Eitan, Almomani) Jordan University of Science and Technology and from the Department of Pathology and Microbiology (Al-Khatib), Jordan University of Science and Technology, Irbid, Jordan.

Received 7th October 2020. Accepted 21st December 2020.

Address correspondence and reprint request to: Dr. Laith N. AL-Eitan, Associate Professor, Department of Biotechnology \& Genetic Engineering, Faculty of Science and Arts, Jordan University of Science and Technology, Irbid, Jordan. E-mail: Ineitan@just.edu.jo

ORCID: https://orcid.org/0000-0003-0064-0190

$H$ elicobacter pylori (H. pylori) is one of the major causes of gastric and duodenal ulcers, chronic gastritis, and gastric carcinomas. ${ }^{1,2}$ The clinical diagnosis of an infection with $H$. pylori is extremely complicated and affected by host and microbial influences. ${ }^{3}$ The diagnosis outcome of $H$. pylori is influenced by many factors including the genetic composition of the infectious strains, the genetic profile of the patients, and particularly the differences in the patients immune system. ${ }^{1,3,4}$ Various cytokine responses to the gastric mucosal inflammation induced by $H$. pylori seem to paly an important role in the patient diagnosis outcome, such as the gastric cancer (GC) and different gastric diseases. ${ }^{2}$ 
However, the association between the variations in the host cytokines genes and the vulnerability to $H$. pylori infections and the extent of the clinical outcome has not been analyzed perfectly. ${ }^{5,6}$ It is recognized that the ethnic diversity of the population is a major element correlated with the frequency variability of many markers and thus influences the vulnerability and intensity of $H$. pylori infection. ${ }^{7,8}$ Several studies have shown that IL-1 $\beta$ is a crucial factor that induces and amplifies inflammatory responses by influencing the levels of gastric mucosal cytokines. ${ }^{9}$

The function of the host genetic factors involved in $H$. pylori infections has been proved., ${ }^{5,6}$ The nucleotidebinding oligomerization domain (NOD)-like receptors (namely, NOD1 and NOD2) have been identified as key factors of chronic inflammation caused by $H$. pylori infections and are important to the clinical outcome of H. pylori infections. ${ }^{6,7}$ Several studies have demonstrated that the genetic variations found in the NOD1 and NOD2 genes among different ethnicities are attributed to different diagnosis manifestations of $H$. pylori infections and are associated with risk of the GC.7,8 Reports have also revealed that there is a relationship between the up-regulation of several inflammatory response genes (for example, TNF- $\alpha$, IL-8, IL-10, IL-1 $\beta$, and IL-1RN) and $H$. pylori infection. ${ }^{9}$ To date, triple therapy involving a mixture of 2 antibiotics (for example, imidazole, amoxicillin clarithromycin, or metronidazole) and one proton pump inhibitor (PPI) (for example, lansoprazole, pantoprazole, omeprazole, or esomeprazole) has shown the best outcome in the treatment of $H$. pylori infection..$^{5,10,11}$ However, the failure rate of this therapy has increased up to $30 \%$ due to several factors, including weak compliance, high bacterial load, antibiotic resistance, high gastric acidity, cytochrome P450 2C19 (CYP2C19) genetic polymorphisms, leading to a reduction in eradication rate of $H$. pylori. ${ }^{10,12}$ The metabolism of PPI is mediated by CYP2C19 in the liver. ${ }^{13}$ Proton pump inhibitors (PPIs) also have a key role in improving the therapeutic efficiency of antibiotics for treatment of $H$. pylori infections and possess direct anti- $H$. pylori activity. ${ }^{10}$ Additionally, PPIs can increase the efficacy of the antibiotic. ${ }^{10-12}$ The genetic polymorphisms of

Disclosure. Authors have no conflict of interests, and the work was not supported or funded by any drug company. This study was funded by Jordan University of Science and Technology, Irbid, Jordan (R\#: 148/2017). the CYP2C19 gene that are considered as a main factor influencing antibiotic resistance among $H$. pylori strains, found to affect both the pharmacokinetic and pharmacodynamic characteristics of PPIs. ${ }^{10,13}$ Therefore, the current research aimed to study the genetic correlation between $H$. pylori infections and peptic ulcers in the Jordanian Arab population and different candidate gene polymorphisms within CYP2C19, TNF- $\alpha$, NOD1, NOD2, and PPAR $\gamma$ genes.

Methods. This retrospective cross-sectional study was conducted to examine the association between a number of genetic polymorphisms of several candidate genes (CYP2C19, TNF- $\alpha$, NOD1, NOD2, and PPAR $\gamma$ ) and the severity of $H$. pylori infections and peptic ulcers (PU). Human ethics approval was obtained from Jordan University of Science and Technology, Irbid, Jordan. All individuals participated in this study signed a written informed consent before sample collection. This study was also performed in accordance with the principles of the Declaration of Helsinki.

A total of 217 formalin fixed paraffin impeded (FFPE) tissues from controls with negative $H$. pylori infections and 251 from Jordanian Arab peptic ulcer patients with $H$. pylori infections were used. All participants visited the endoscopy clinic during 2014 to 2018 in the King Abdullah University Hospital (KAUH), Irbid, Jordan. Clinical and demographic data were collected using the computerized systems in the KAUH. Patients were included if they are of Arab descent and have a confirmed H. pylori infection by a consultant based on symptoms and clinical data. Patients were classified according to the Updated Sydney Classification on H. pylori chronic gastritis into 2 groups (active/acute and inactive/ chronic) based on the presence of mononuclear cells (lymphocytes) and neutrophils (multinuclear cells) in the microscopic histopathological analysis. In addition, the severity of the $H$. pylori infection was based on the number of multinuclear cells and classified into 3 groups: mild, moderate, and severe.

DNA analysis. The DNeasy Blood \& Tissue Kit (Qiagen Ltd., West Sussex, UK) was used for genomic DNA extraction from FFPE tissue that was taken from the patients who visited the endoscopy clinics. The single nuclotide polymorphisms (SNPs) were genotyped using the Sequenom MassARRAY ${ }^{\odot}$ system (iPLEX GOLD) (Sequenom, San Diego, CA, USA) at the Australian Genome Research Facility (AGRF, Melbourne Node, Melbourne, Australia) for CYP2C19 (rs17882687, rs6413438, rs4986893, and rs17879685), TNF- $\alpha$ (rs1799964, rs1800629, and rs361525), NOD1 (rs2075820, rs2907749, and rs7789045), NOD2 
(rs1861759 and rs3135500), IL-1 $\beta$ (rs16944 and rs1143627), and PPAR $\gamma$ (rs10865710, rs1801282, rs3856806, and rs13306747) genes.

Statistical analysis. The genotypic distribution and the correlation between the CYP2C19 (rs17882687, rs6413438, rs4986893, and rs17879685), TNF- $\alpha$ (rs1799964, rs1800629, and rs361525), NOD1 (rs2075820, rs2907749, and rs7789045), NOD2

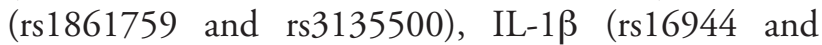
rs1143627), and PPAR $\gamma$ (rs10865710, rs1801282, rs3856806, and rs13306747) polymorphisms and clinical variables (independent variables) were analyzed using the Chi-square test and estimated the risk as an odds ratio with a 95\% confidence interval. The HardyWeinberg equilibrium (HWE) values and the genetic haplotype association were assessed using the SNPStats Web Tool (https://www.snpstats.net/start.htm). Finally, the Statistical Package for Social Sciences IBM SPSS Statistics for Windows Version 25 (IBM Corp., Armonk, N.Y., USA) was used to identify the genotypic, allelic, and clinical data associations with $H$. pylori infection.

Results. Demographic and clinical characteristics of 251 peptic ulcer patients caused by $H$. pylori are presented in Table 1. The average age of the patients was $42.78 \pm 17.3$ years for males and $41.57 \pm 16.4$ for females. A significant difference was found between gender and the activity of $H$. pylori with a $p=0.032$, where the percentage of inactive $H$. pylori in female patients was 4 times that of male patients. These results confirmed previous studies that there were gender differences in the response to $H$. pylori infections that may lead to variances in inflammation scores, activity, intestinal metaplasia, and different incidences of gastric cancer between men and women. Many scientists have hypothesized that this variation in response is due to basic biological differences between men and women, including hormones-particularly estrogenand differences in lifestyles, such as diet and smoking. Table 2 reveals the MAF of the studied polymorphisms (rs17882687, rs6413438, rs4986893, and rs17879685 within the CYP2C19 gene, rs1799964, rs1800629, and rs361525 within the TNF- $\alpha$ gene, rs2075820, rs2907749, and rs7789045 within the NOD1 gene, rs1861759 and rs3135500 within the NOD2 gene, rs16944 and rs1143627 within the IL-1 $\beta$ gene, and rs10865710, rs 1801282 , rs3856806, and rs 13306747 within the PPAR $\gamma$ gene) and their location on the genomic DNA as well as the HWE $p$-value.

All studied genotypes were in HWE $(p>0.05)$, so it is suggested that the population was derived from the same as the Mendelian population, except for 6 SNPs.
Four polymorphisms were found as monomorphic (rs6413438, rs4986893, and rs17879685 in the CYP2C19 gene and rs13306747 in the PPAR $\gamma$ gene). Morover, genotypic and allelic distribution of these SNPs are shown in Table 3 and revealed no significant differences in the SNP distribution frequencies of the CYP2C19, TNF- $\alpha$, NOD2, and PPAR $\gamma$ genes between patients and controls.

For the NOD1 polymorphisms, the only SNP that showed a significant difference in genotype frequencies was rs2075820 (C>T) between healthy subjects and peptic ulcer patients $(p<0.05) ; 43.5 \%$ (TT), $44.8 \%$ (CT), and $11.7 \%$ (CC) in patients compared to $29.2 \%$

Table 1 - Demographic and clinical details of PU patients (251) in comparison with gender.

\begin{tabular}{|c|c|c|c|}
\hline \multirow[t]{2}{*}{ Clinical data } & \multicolumn{2}{|c|}{ Gender } & \multirow[t]{2}{*}{$P$-value* } \\
\hline & Male & Female & \\
\hline Age & $42.78 \pm 17.3$ & $41.57 \pm 16.4$ & 0.57 \\
\hline $\begin{array}{l}\text { Marital status } \\
\text { Single } \\
\text { Married } \\
\text { Divorced } \\
\text { Widowed }\end{array}$ & $\begin{array}{rr}38 & (15.1) \\
101 & (40.2) \\
1 & (0.4) \\
& 0(0)\end{array}$ & $\begin{array}{rr}30 & (12.0) \\
80 & (31.9) \\
0 & (0.0) \\
1 & (0.4)\end{array}$ & 0.03 \\
\hline $\begin{array}{l}\text { Helicobacter pylor } \\
\text { Yes } \\
\text { No }\end{array}$ & $\begin{array}{r}104(41.1) \\
7 \quad(2.8)\end{array}$ & $\begin{array}{r}120(47.8) \\
20 \quad(8.0)\end{array}$ & 0.032 \\
\hline $\begin{array}{l}\text { Other diseases } \\
\text { No } \\
\text { Yes }\end{array}$ & $\begin{array}{l}61(24.3) \\
47(18.7)\end{array}$ & $\begin{array}{l}83(33.1) \\
58(23.1)\end{array}$ & 0.58 \\
\hline $\begin{array}{l}\text { Duodenal ulcer } \\
\text { No } \\
\text { Yes }\end{array}$ & $\begin{array}{l}92(36.7) \\
19 \quad(7.6)\end{array}$ & $\begin{array}{r}122(48.6) \\
18 \quad(7.2)\end{array}$ & 0.22 \\
\hline $\begin{array}{l}\text { Esophagitis } \\
\text { No } \\
\text { Yes }\end{array}$ & $\begin{array}{lr}89 & (35.5) \\
22 & (8.8)\end{array}$ & $\begin{array}{r}115(45.8) \\
25(10.0)\end{array}$ & 0.23 \\
\hline $\begin{array}{l}\text { Chronicity } \\
\text { Yes } \\
\text { No }\end{array}$ & $\begin{array}{r}110(43.8) \\
1 \quad(0.4)\end{array}$ & $\begin{array}{r}139(55.4) \\
1 \quad(0.4)\end{array}$ & 0.35 \\
\hline $\begin{array}{l}\text { Proton pump inhi } \\
\text { No } \\
\text { Lansoprazole } \\
\text { Esomeprazole } \\
\text { Omeprazole } \\
\text { Two or three }\end{array}$ & $\begin{array}{rr}1 & (0.4) \\
86 & (34.3) \\
0 & (0.0) \\
1 & (0.4) \\
43 & (9.2)\end{array}$ & $\begin{array}{rr}7 & (2.8) \\
97 & (38.3) \\
3 & (1.2) \\
1 & (0.4) \\
32 & (12.8)\end{array}$ & 0.488 \\
\hline $\begin{array}{l}\text { Antibiotics } \\
\text { No } \\
\text { Amoxicillin } \\
\text { Clarithromycin } \\
\text { Amoxicillin + } \\
\text { Clarithromycin } \\
\text { Others }\end{array}$ & $\begin{array}{rr}27 & (10.8) \\
5 & (2.0) \\
1 & (0.4) \\
76 & (30.3) \\
2 & (0.8)\end{array}$ & $\begin{array}{rr}33 & (13.1) \\
2 & (0.8) \\
4 & (1.6) \\
97 & (38.6) \\
4 & (1.6)\end{array}$ & 0.65 \\
\hline Valı & $\begin{array}{l}\mathrm{d} \text { as number } \\
\text { considered } \mathrm{s}\end{array}$ & $\begin{array}{l}\text { d percentage } \\
\text { ificant }\end{array}$ & \\
\hline
\end{tabular}


Table 2 - Details of the candidate genes polymorphisms of interest and the minor allele frequency.

\begin{tabular}{|c|c|c|c|c|c|c|c|}
\hline \multirow[t]{2}{*}{ Gene } & \multirow{2}{*}{$\begin{array}{c}\text { Chromosome } \\
\#\end{array}$} & \multirow[t]{2}{*}{ rs numbers } & \multirow{2}{*}{$\begin{array}{l}\text { Minor } \\
\text { allele }\end{array}$} & \multicolumn{2}{|c|}{ Patients } & \multicolumn{2}{|c|}{ Control } \\
\hline & & & & MAF & HWE $p$-value & MAF & HWE $p$-value \\
\hline \multirow[t]{4}{*}{ CYP2C19 } & $10 \mathrm{q} 23.33$ & rs 17882687 & $\mathrm{C}$ & 0.01 & 1 & 0.01 & 1 \\
\hline & & rs6413438 & $\mathrm{T}$ & 0 & NA & 0 & NA \\
\hline & & rs 4986893 & $\mathrm{C}$ & 0 & NA & 0 & NA \\
\hline & & rs 17879685 & $\mathrm{~T}$ & 0 & NA & 0 & NA \\
\hline \multirow[t]{3}{*}{ TNF- $\alpha$} & $6 \mathrm{p} 21.33$ & rs1799964 & $\mathrm{C}$ & 0.19 & 0.68 & 0.17 & 1 \\
\hline & & rs1800629 & A & 0.11 & 1 & 0.12 & 1 \\
\hline & & rs361525 & A & 0.07 & 0.27 & 0.06 & 0.58 \\
\hline \multirow[t]{3}{*}{ NOD1 } & $7 \mathrm{p} 14.3$ & rs 2075820 & $\mathrm{~T}$ & 0.34 & 1 & 0.41 & 0.00042 \\
\hline & & rs2907749 & G & 0.33 & 0.24 & 0.32 & 0.046 \\
\hline & & rs7789045 & $\mathrm{T}$ & 0.45 & 0.44 & 0.4 & 0.28 \\
\hline \multirow[t]{2}{*}{ NOD2 } & $16 \mathrm{q} 12.1$ & rs1861759 & G & 0.42 & 0.5 & 0.42 & Т0.17 \\
\hline & & rs3135500 & A & 0.46 & 0.88 & 0.44 & 0.26 \\
\hline \multirow[t]{2}{*}{ IL-1 $\beta$} & $2 \mathrm{q} 14.1$ & rs16944 & A & 0.36 & 1 & 0.43 & 1 \\
\hline & & rs1143627 & G & 0.38 & 0.48 & 0.31 & 0.87 \\
\hline \multirow[t]{4}{*}{$\operatorname{PPAR} \gamma$} & $3 \mathrm{p} 25.2$ & rs10865710 & G & 0.17 & 0.35 & 0.18 & 0.62 \\
\hline & & rs1801282 & G & 0.03 & 1 & 0.041 & 0.23 \\
\hline & & rs3856806 & $\mathrm{T}$ & 0.04 & 1 & 0.05 & 0.098 \\
\hline & & rs 13306747 & $\mathrm{C}$ & 0 & NA & 0 & NA \\
\hline
\end{tabular}

(TT), 61.3\% (TC), and $9.5 \%$ (CC) in controls. The homozygous (CC) genotype frequency was notably increased in peptic patients compared to the control group and the opposite for the heterozygous (TC) frequency, so the homozygous genotype (CC) could have an increased risk of peptic ulcer (odd ratio: 0.49, $p=0.0046$ ), but this polymorphism and the remaining studied SNPs showed an inconsiderable association of allele frequency between patients and healthy individuals.

Table 4 illustrates the correlation between the activity of $H$. pylori and the candidate polymorphisms in peptic ulcer patients. The data showed no significant association between the activity of $H$. pylori (active/acute and inactive/chronic) and the polymorphisms of the studied genes with a $p>0.05$. Moreover, the relationship between the studied polymorphisms and the severity of the $H$. pylori infection (mild, moderate, or severe) in peptic ulcer patients are shown in Table 5. The statistical analysis of the data revealed there was no important association between the severity of the $H$. pylori infection and these studied SNPs with a $p>0.05$.
Discussion. Helicobacter pylori is one of the predominant human pathogens with a worldwide infection rate of more than $50 \% .{ }^{14-16}$ The treatments for $H$. pylori elimination consist of PPIs and antimicrobial drugs (namely, amoxicillin, clarithromycin, and metronidazole). ${ }^{17,18}$ Proton pump inhibitors are mainly metabolized by CYP2C19, which is highly polymorphic. ${ }^{10,11,19}$ Many recent studies have reported that the CYP2C19 genotypes have variable effectiveness of PPIs and imperative roles in peptic ulcer healing. ${ }^{20,21}$ The distributions of the CYP2C19 polymorphisms vary among different populations..$^{22,23}$ This study was conducted to explore the impacts of genetic polymorphisms of different candidate genes (CYP2C19 [rs17882687, rs6413438, rs4986893, and rs17879685], TNF- $\alpha$ [rs1799964, rs1800629, and rs361525], NOD1 [rs2075820, rs2907749, and rs7789045], NOD2 [rs1861759 and rs3135500], IL-1 $\beta$ [rs16944 and rs1143627], and PPAR $\gamma$ [rs10865710, rs1801282, rs3856806, and rs13306747]) on the risk of gastric lesions in a Jordanian Arab descent patients. 
Table 3 - Distribution of the genotypes of the studied genes among cases and controls.

\begin{tabular}{|c|c|c|c|c|}
\hline Gene/rs numbers/models & Control (\%) & PUD patients (\%) & Odd ratio $(95 \% \mathrm{CI})$ & P-value \\
\hline \multicolumn{5}{|l|}{$T N F-\alpha$} \\
\hline \multicolumn{5}{|l|}{$r s 1799964$} \\
\hline TT / TC / CC & $69 / 28.4 / 2.5$ & $65.7 / 30.2 / 4$ & $1 / 1.12 / 1.67$ & 0.59 \\
\hline $\mathrm{TT} / \mathrm{TC}+\mathrm{CC}$ & $69 / 31$ & $65.7 / 34.3$ & $1 / 1.16$ & 0.46 \\
\hline $\mathrm{TT}+\mathrm{TC} / \mathrm{CC}$ & $97.5 / 2.5$ & $96 / 4$ & $1 / 1.61$ & 0.38 \\
\hline $\mathrm{TT}+\mathrm{CC} / \mathrm{TC}$ & $71.6 / 28.4$ & $69.8 / 30.2$ & $1 / 1.09$ & 0.68 \\
\hline \multicolumn{5}{|l|}{$r s 1800629$} \\
\hline $\mathrm{GG} / \mathrm{GA} / \mathrm{AA}$ & $78 / 20.6 / 1.4$ & $79.5 / 19.3 / 1.2$ & $1 / .92 / .84$ & 0.92 \\
\hline $\mathrm{GG} / \mathrm{GA}+\mathrm{AA}$ & $78 / 22$ & $79.5 / 20.5$ & $1 / 0.91$ & 0.96 \\
\hline $\mathrm{GG}+\mathrm{GA} / \mathrm{AA}$ & $98.6 / 1.4$ & $98.8 / 1.2$ & $1 / .85$ & 0.85 \\
\hline \multirow{2}{*}{\multicolumn{5}{|c|}{$\begin{array}{l}\text { GG + AA/GA } \\
r s 361525\end{array}$}} \\
\hline & & & & \\
\hline $\mathrm{GG} / \mathrm{GA} / \mathrm{AA}$ & $87.6 / 11.9 / .5$ & $87.8 / 11.4 / 0.8$ & $1 / 0.96 / 1.64$ & 0.91 \\
\hline $\mathrm{GG} / \mathrm{GA}+\mathrm{AA}$ & $87.6 / 12.4$ & $87.8 / 12.2$ & $1 / 0.98$ & 0.95 \\
\hline $\mathrm{GG}+\mathrm{GA} / \mathrm{AA}$ & $99.5 / 0.5$ & $99.2 / 0.8$ & $1 / 1.65$ & 0.68 \\
\hline $\mathrm{GG}+\mathrm{AA} / \mathrm{GA}$ & $99.2 / 0.8$ & $88.6 / 11.4$ & $1 / 0.95$ & 0.87 \\
\hline \multicolumn{5}{|l|}{ NOD1 } \\
\hline \multicolumn{5}{|l|}{$r s 2075820$} \\
\hline $\mathrm{CC} / \mathrm{CT} / \mathrm{TT}$ & $29.2 / 61.3 / 9.5$ & $43.5 / 44.8 / 11.7$ & $1 / 0.49 / 0.82$ & 0.0046 \\
\hline $\mathrm{CC} / \mathrm{TC}+\mathrm{TT}$ & $29.2 / 70.8$ & $43.5 / 56.5$ & $1 / 0.53$ & 0.0035 \\
\hline $\mathrm{CC}+\mathrm{TC} / \mathrm{TT}$ & $90.5 / 9.5$ & $88.3 / 11.7$ & $1 / 1.25$ & 0.5 \\
\hline \multirow{2}{*}{\multicolumn{5}{|c|}{$r s 2907749$}} \\
\hline & & & & \\
\hline GG/ GA / AA & $43.5 / 49.7 / 6.8$ & $42.9 / 47.9 / 9.2$ & $1 / 0.98 / 1.36$ & 0.66 \\
\hline $\mathrm{GG} / \mathrm{GA}+\mathrm{AA}$ & $43.5 / 56.5$ & $42.9 / 57.1$ & $1 / 1.02$ & 0.91 \\
\hline $\mathrm{GG}+\mathrm{GA} / \mathrm{AA}$ & $93.2 / 6.8$ & $90.8 / 9.2$ & $1 / 1.38$ & 0.37 \\
\hline $\mathrm{GG}+\mathrm{AA} / \mathrm{GA}$ & $50.3 / 49.7$ & $52.1 / 47.9$ & $1 / 0.93$ & 0.71 \\
\hline \multicolumn{5}{|l|}{$r s 7789045$} \\
\hline $\mathrm{TT} / \mathrm{AT} / \mathrm{AA}$ & $34.2 / 51.9 / 13.8$ & $29.2 / 52.1 / 18.8$ & $1 / 1.18 / 1.59$ & 0.3 \\
\hline $\mathrm{TT} / \mathrm{AT}+\mathrm{AA}$ & $34.2 / 65.8$ & $29.2 / 70.8$ & $1 / 1.27$ & 0.27 \\
\hline $\mathrm{TT}+\mathrm{AT} / \mathrm{AA}$ & $86.2 / 13.8$ & $81.2 / 18.8$ & $1 / 1.44$ & 0.17 \\
\hline $\mathrm{TT}+\mathrm{AA} / \mathrm{AT}$ & $48.1 / 51.9$ & $47.9 / 52.1$ & $1 / 1.01$ & 0.98 \\
\hline \multicolumn{5}{|l|}{ NOD2 } \\
\hline \multicolumn{5}{|l|}{$r s 1861759$} \\
\hline TT / GT / GG & $30.6 / 54.4 / 15$ & $35.1 / 46.5 / 18.4$ & $1 / 0.74 / 1.07$ & 0.27 \\
\hline TT / GT + GG & $30.6 / 69.4$ & $35.1 / 64.9$ & $1 / 0.81$ & 0.33 \\
\hline $\mathrm{TT}+\mathrm{GT} / \mathrm{GG}$ & $85 / 15$ & $81.6 / 18.4$ & $1 / 1.28$ & 0.36 \\
\hline $\mathrm{TT}+\mathrm{GG} / \mathrm{GA}$ & $45.6 / 54.4$ & $53.5 / 46.5$ & $1 / 0.73$ & 0.11 \\
\hline \multicolumn{5}{|l|}{$r s 3135500$} \\
\hline $\mathrm{GG} / \mathrm{GA} / \mathrm{AA}$ & $28.5 / 54.4 / 17.1$ & $29.2 / 48.9 / 21.7$ & $1 / 0.87 / 1.23$ & 0.49 \\
\hline $\mathrm{GG} / \mathrm{GA}+\mathrm{AA}$ & $28.5 / 71.5$ & $29.4 / 70.6$ & $1 / 0.95$ & 0.85 \\
\hline $\mathrm{GG}+\mathrm{GA} / \mathrm{AA}$ & $82.9 / 17.1$ & $78.3 / 21.7$ & $1 / 1.34$ & 0.31 \\
\hline $\mathrm{GG}+\mathrm{AA} / \mathrm{GA}$ & $45.6 / 54.4$ & $51.1 / 48.9$ & $1 / 0.8$ & 0.63 \\
\hline IL- $1 \beta$ & & & & \\
\hline rs 16944 & & & & \\
\hline $\mathrm{GG} / \mathrm{GA} / \mathrm{AA}$ & $32.2 / 49.4 / 18.4$ & $41.1 / 46.1 / 12.8$ & $1 / 0.73 / 0.54$ & 0.12 \\
\hline $\mathrm{GG} / \mathrm{GA}+\mathrm{AA}$ & $32.2 / 67.8$ & $41.1 / 58.9$ & $1 / 0.68$ & 0.069 \\
\hline $\mathrm{GG}+\mathrm{GA} / \mathrm{AA}$ & $81.6 / 18.4$ & $87.2 / 12.8$ & $1 / 0.65$ & 0.13 \\
\hline $\mathrm{GG}+\mathrm{AA} / \mathrm{GA}$ & $50.6 / 49.4$ & $53.9 / 46.1$ & $1 / 0.88$ & 0.51 \\
\hline rs1143627 & & & & \\
\hline $\mathrm{GG} / \mathrm{GA} / \mathrm{AA}$ & $31.1 / 50.3 / 18.6$ & $37.6 / 49.6 / 12.8$ & $1 / 0.81 / 0.57$ & 0.18 \\
\hline $\mathrm{GG} / \mathrm{GA}+\mathrm{AA}$ & $31.1 / 68.9$ & $37.6 / 62.4$ & $1 / 0.75$ & 0.11 \\
\hline $\mathrm{GG}+\mathrm{GA} / \mathrm{AA}$ & $81.4 / 18.6$ & $87.2 / 12.8$ & $1 / 0.64$ & 0.89 \\
\hline $\mathrm{GG}+\mathrm{AA} / \mathrm{GA}$ & $49.7 / 50.3$ & $50.4 / 49.6$ & $1 / 0.97$ & 0.071 \\
\hline $\operatorname{PPAR} \gamma$ & & & & \\
\hline$r s 16944$ & & & & \\
\hline $\mathrm{GG} / \mathrm{GC} / \mathrm{CC}$ & $32.2 / 49.4 / 18.4$ & $41.1 / 46.1 / 12.8$ & $1 / 0.73 / 0.54$ & 0.12 \\
\hline $\mathrm{GG} / \mathrm{GC}+\mathrm{CC}$ & $32.2 / 67.8$ & $41.1 / 58.9$ & $1 / 0.68$ & 0.069 \\
\hline $\mathrm{GG}+\mathrm{GC} / \mathrm{CC}$ & $81.6 / 18.4$ & $87.2 / 12.8$ & $1 / 0.65$ & 0.13 \\
\hline $\mathrm{GG}+\mathrm{CC} / \mathrm{GC}$ & $50.6 / 49.4$ & $53.9 / 46.1$ & $1 / 0.88$ & 0.51 \\
\hline$r s 1801282$ & & & & \\
\hline CC / CG / GG & $31.1 / 50.3 / 18.6$ & $37.6 / 49.6 / 12.8$ & $1 / 0.81 / 0.57$ & 0.18 \\
\hline $\mathrm{CC} / \mathrm{CG}+\mathrm{GG}$ & $31.1 / 68.9$ & $37.6 / 62.4$ & $1 / 0.75$ & 0.11 \\
\hline $\mathrm{CC}+\mathrm{CG} / \mathrm{GG}$ & $81.4 / 18.6$ & $87.2 / 12.8$ & $1 / 0.64$ & 0.89 \\
\hline $\mathrm{CC}+\mathrm{GG} / \mathrm{CG}$ & $49.7 / 50.3$ & $50.4 / 49.6$ & $1 / 0.97$ & 0.071 \\
\hline$r s 3856806$ & & & & \\
\hline $\mathrm{CC} / \mathrm{CT} / \mathrm{TT}$ & $90.5 / 8.6 / 1$ & $92 / 8 / 0.0$ & $1 / 0.91 / 0.0$ & 0.2 \\
\hline $\mathrm{CC} / \mathrm{CT}+\mathrm{TT}$ & $90.5 / 9.5$ & $92 / 8$ & $1 / 0.82$ & $\mathrm{NA}^{* *}$ \\
\hline $\mathrm{CC}+\mathrm{CT} / \mathrm{TT}$ & $99 / 1$ & $100 / 0.0$ & $1 / 0.0$ & 0.076 \\
\hline $\mathrm{CC}+\mathrm{TT} / \mathrm{CT}$ & $91.4 / 8.6$ & $92 / 8$ & $1 / 0.92$ & 0.81 \\
\hline
\end{tabular}

MAF: minor allele frequency, HWE: hardy weinberg equilibrium $p>0.05$, NA: not available, PUD: peptic ulcer disease 
Table 4 - The effect of the genotype distribution of studied SNPs on the activity of Helicobacter pylori infected Jordanian patients.

\begin{tabular}{|c|c|c|c|c|}
\hline \multirow[t]{2}{*}{ Gene/rs numbers/models } & \multicolumn{2}{|c|}{ Activity } & \multirow[t]{2}{*}{ Odd ratio $(95 \% \mathrm{CI})$} & \multirow[t]{2}{*}{$P$-value } \\
\hline & Yes & No & & \\
\hline \multicolumn{5}{|l|}{$T N F-\alpha$} \\
\hline $\mathrm{TT} / \mathrm{TC} / \mathrm{CC}$ & $64.4 / 31.1 / 4.6$ & $74.1 / 25.1 / 0$ & $1 / 1.38 / \mathrm{NA}$ & 0.24 \\
\hline $\mathrm{TT} / \mathrm{TC}+\mathrm{CC}$ & $64.4 / 35.6$ & $74.1 / 25.9$ & $1 / 1.58$ & 0.31 \\
\hline $\mathrm{TT}+\mathrm{TC} / \mathrm{CC}$ & $95.4 / 4.6$ & $100 / 0$ & $1 / \mathrm{NA}$ & 0.12 \\
\hline $\mathrm{TT}+\mathrm{CC} / \mathrm{TC}$ & $69 / 31$ & $74.1 / 25.9$ & $1 / 1.29$ & 0.2 \\
\hline \multicolumn{5}{|l|}{$r s 1800629$} \\
\hline GG/ GA / AA & $80 / 18.6 / 1.4$ & $74.1 / 25.9$ & $1 / 0.66 / \mathrm{NA}$ & 0.49 \\
\hline $\mathrm{GG} / \mathrm{GA}+\mathrm{AA}$ & $80 / 20$ & $74.1 / 25.9$ & $1 / 0.71$ & 0.48 \\
\hline $\mathrm{GG}+\mathrm{GA} / \mathrm{AA}$ & $98.6 / 1.4$ & $100 / 0$ & $1 / \mathrm{NA}$ & 0.4 \\
\hline $\mathrm{GG}+\mathrm{AA} / \mathrm{GA}$ & $81.4 / 18.6$ & $74.1 / 25.9$ & $1 / 0.65$ & 0.38 \\
\hline \multicolumn{5}{|l|}{$r s 361525$} \\
\hline GG/ GA / AA & $86.4 / 12.4 / 0.9$ & $96.3 / 3.7 / 0$ & $1 / 3.73 / \mathrm{NA}$ & 0.24 \\
\hline $\mathrm{GG} / \mathrm{GA}+\mathrm{AA}$ & $86.6 / 13.4$ & $96.3 / 3.7$ & $1 / 4.01$ & 0.1 \\
\hline $\mathrm{GG}+\mathrm{GA} / \mathrm{AA}$ & $99.1 / 0.9$ & $100 / 0$ & $1 / \mathrm{NA}$ & 0.49 \\
\hline $\mathrm{GG}+\mathrm{AA} / \mathrm{GA}$ & $87.6 / 12.4$ & $96.3 / 3.7$ & $1 / 3.69$ & 0.13 \\
\hline \multicolumn{5}{|l|}{ NOD1 } \\
\hline \multicolumn{5}{|l|}{$r s 2075820$} \\
\hline $\mathrm{CC} / \mathrm{CT} / \mathrm{TT}$ & $42.4 / 46 / 11.6$ & $52.2 / 34.8 / 13$ & $1 / 1.62 / 1.1$ & \\
\hline $\mathrm{CC} / \mathrm{TC}+\mathrm{TT}$ & $42.4 / 57.6$ & $52.2 / 47.8$ & $1.0 / 1.48$ & 0.37 \\
\hline $\mathrm{CC}+\mathrm{TC} / \mathrm{TT}$ & $88.4 / 11.6$ & $87 / 13$ & $1.0 / 0.88$ & 0.84 \\
\hline $\mathrm{CC}+\mathrm{TT} / \mathrm{TC}$ & $54 / 46$ & $65.2 / 34.8$ & $1.0 / 1.59$ & 0.3 \\
\hline \multicolumn{5}{|l|}{$r s 2907749$} \\
\hline GG/ GA / AA & $43.4 / 49.1 / 7.5$ & $42.3 / 38.5 / 19.2$ & $1.0 / 1.24 / 0.38$ & 0.18 \\
\hline $\mathrm{GG} / \mathrm{GA}+\mathrm{AA}$ & $43.4 / 56.4$ & $42.3 / 57.7$ & $1.0 / 0.96$ & 0.92 \\
\hline $\mathrm{GG}+\mathrm{GA} / \mathrm{AA}$ & $92.5 / 7.5$ & $80.8 / 19.2$ & $1.0 / 0.34$ & 0.076 \\
\hline $\mathrm{GG}+\mathrm{AA} / \mathrm{GA}$ & $50.9 / 49.1$ & $61.5 / 38.5$ & $1.0 / 1.54$ & 0.3 \\
\hline \multicolumn{5}{|l|}{ rs 7789045} \\
\hline $\mathrm{TT} / \mathrm{AT} / \mathrm{AA}$ & $28.9 / 52.6 / 18.5$ & $25.9 / 51.9 / 22.2$ & $1.0 / 0.91 / 0.75$ & 0.88 \\
\hline $\mathrm{TT} / \mathrm{AT}+\mathrm{AA}$ & $28.9 / 71.1$ & $25.9 / 74.1$ & $1.0 / 0.86$ & 0.74 \\
\hline $\mathrm{TT}+\mathrm{AT} / \mathrm{AA}$ & $81.5 / 18.5$ & $77.8 / 22.2$ & $1.0 / 0.79$ & 0.65 \\
\hline $\mathrm{TT}+\mathrm{AA} / \mathrm{AT}$ & $47.7 / 52.6$ & $48.1 / 51.9$ & $1.0 / 1.03$ & 0.94 \\
\hline \multicolumn{5}{|l|}{ NOD2 } \\
\hline \multicolumn{5}{|l|}{$r s 1861759$} \\
\hline TT / GT / GG & $34.2 / 47.5 / 18.3$ & $41.7 / 41.7 / 16.7$ & $1.0 / 1.39 / 1.34$ & 0.77 \\
\hline $\mathrm{TT} / \mathrm{GT}+\mathrm{GG}$ & $34.2 / 65.8$ & $41.7 / 58.3$ & $1.0 / 1.38$ & 0.47 \\
\hline $\mathrm{TT}+\mathrm{GT} / \mathrm{GG}$ & $81.7 / 18.3$ & $83.3 / 16.7$ & $1.0 / 1.12$ & 0.84 \\
\hline $\mathrm{TT}+\mathrm{GG} / \mathrm{GA}$ & $52.5 / 47.5$ & $58.3 / 41.5$ & $1.0 / 1.27$ & 0.59 \\
\hline \multicolumn{5}{|l|}{$r s 3135500$} \\
\hline GG/ GA / AA & $29.4 / 47.9 / 22.7$ & $29.4 / 58.8 / 11.8$ & $1.0 / 0.81 / 1.93$ & 0.51 \\
\hline $\mathrm{GG} / \mathrm{GA}+\mathrm{AA}$ & $29.4 / 70.5$ & $29.4 / 70.6$ & $1.0 / 1.0$ & 1 \\
\hline $\mathrm{GG}+\mathrm{GA} / \mathrm{AA}$ & $77.3 / 22.7$ & $88.2 / 11.8$ & $1.0 / 2.0$ & 0.27 \\
\hline $\mathrm{GG}+\mathrm{AA} / \mathrm{GA}$ & $52.1 / 47.9$ & $41.2 / 58.8$ & $1.0 / 0.64$ & 0.39 \\
\hline \multicolumn{5}{|l|}{$I L-1 \beta$} \\
\hline \multicolumn{5}{|l|}{ rs 16944} \\
\hline GG/ GA / AA & $41.8 / 45.4 / 12.9$ & $33.3 / 54.2 / 12.5$ & $1.0 / 0.67 / 0.82$ & 0.69 \\
\hline $\mathrm{GG} / \mathrm{GA}+\mathrm{AA}$ & $41.8 / 58.2$ & $33.3 / 66.7$ & $1.0 / 0.7$ & 0.42 \\
\hline $\mathrm{GG}+\mathrm{GA} / \mathrm{AA}$ & $87.1 / 12.9$ & $87.5 / 12.5$ & $1.0 / 1.04$ & 0.96 \\
\hline $\mathrm{GG}+\mathrm{AA} / \mathrm{GA}$ & $54.6 / 45.4$ & $45.8 / 54.2$ & $1.0 / 0.7$ & 0.42 \\
\hline$r s 1143627$ & & & & \\
\hline GG/ GA / AA & $38.2 / 49.2 / 12.6$ & $30.8 / 53.9 / 15.4$ & $1.0 / 0.74 / 0.66$ & 0.74 \\
\hline $\mathrm{GG} / \mathrm{GA}+\mathrm{AA}$ & $38.2 / 61.8$ & $30.8 / 69.2$ & $1 / 0.72$ & 0.46 \\
\hline $\mathrm{GG}+\mathrm{GA} / \mathrm{AA}$ & $87.4 / 12.6$ & $84.6 / 15.4$ & $1.0 / 0.79$ & 0.69 \\
\hline $\mathrm{GG}+\mathrm{AA} / \mathrm{GA}$ & $50.8 / 49.2$ & $46.1 / 53.9$ & $1.0 / 0.83$ & 0.66 \\
\hline $\begin{array}{l}P P A R \gamma \\
\quad r s 10865710\end{array}$ & & & & \\
\hline $\mathrm{GG} / \mathrm{GC} / \mathrm{CC}$ & $68.2 / 29.9 / 1.9$ & $70.4 / 29.6 / 0$ & $1 / 104 / \mathrm{NA}$ & \\
\hline $\mathrm{GG} / \mathrm{GC}+\mathrm{CC}$ & $68.2 / 31.8$ & 70.4 / 29.6 & $1 / 1.11$ & 0.82 \\
\hline $\mathrm{GG}+\mathrm{GC} / \mathrm{CC}$ & $98.1 / 1.9$ & $100 / 0$ & $1 / \mathrm{NA}$ & 0.32 \\
\hline $\mathrm{GG}+\mathrm{CC} / \mathrm{GC}$ & $70.1 / 29.9$ & $70.4 / 29.6$ & $1 / 1.01$ & 0.98 \\
\hline
\end{tabular}


Table 5 - The effect of the genotype distribution of studied SNPs on the severity of Helicobacter pylori infected Jordanian patients.

\begin{tabular}{|c|c|c|c|c|}
\hline \multirow{2}{*}{$\begin{array}{l}\text { Gene/rs } \\
\text { numbers/ } \\
\text { genotype }\end{array}$} & \multicolumn{3}{|c|}{ Activity } & \multirow[t]{2}{*}{$P$-value } \\
\hline & Mild & Moderate & Severe & \\
\hline \multicolumn{5}{|l|}{$T N F-\alpha$} \\
\hline TT & $44(20.0)$ & $73(33.1)$ & 25 (11.3) & \multirow{3}{*}{0.46} \\
\hline TC & $19 \quad(8.5)$ & $33(15.0)$ & 16 & \\
\hline $\mathrm{CC}$ & $2(0.0)$ & $6 \quad(0.3)$ & $2(0.0)$ & \\
\hline \multicolumn{5}{|c|}{ rs1800629 } \\
\hline GG & $53 \quad(2.0)$ & $86(39.8)$ & $34(15.7)$ & \multirow{3}{*}{0.74} \\
\hline GA & 9 (4.2) & $24(11.1)$ & $7 \quad(3.2)$ & \\
\hline $\mathrm{AA}$ & $1 \quad(0.5)$ & $1 \quad(0.5)$ & $1 \quad(0.5)$ & \\
\hline \multicolumn{5}{|c|}{$r s 361525$} \\
\hline GG & $53(24.3)$ & 96 (44.0) & $40(18.3)$ & \multirow{3}{*}{0.75} \\
\hline GA & $9(4.1)$ & $15 \quad(6.9)$ & $3(1.4)$ & \\
\hline $\mathrm{AA}$ & $1 \quad(0.5)$ & $1 \quad(0.5)$ & $\begin{array}{ll}0 & (0.0)\end{array}$ & \\
\hline \multicolumn{5}{|c|}{ NOD1 } \\
\hline$r s 20758$ & & & & \multirow{4}{*}{0.83} \\
\hline TT & $7 \quad(3.5)$ & $12(6.0)$ & $4 \quad(2.0)$ & \\
\hline TC & 31 (15.6) & $31(15.0)$ & $17(8.5)$ & \\
\hline CC & $23(11.6)$ & $45(22.2)$ & $16(8.0)$ & \\
\hline \multicolumn{5}{|c|}{$r s 2907749$} \\
\hline GG & $3 \quad(1.4)$ & $10 \quad(4.7)$ & $3 \quad(1.4)$ & \multirow{3}{*}{0.86} \\
\hline GA & $34(16.0)$ & 53 (24.6) & $48(22.5)$ & \\
\hline AA & $17(8.0)$ & $18 \quad(8.5)$ & $2(1.4)$ & \\
\hline \multicolumn{5}{|c|}{$r s 7789045$} \\
\hline TT & $14 \quad(6.6)$ & $20 \quad(9.4)$ & $5 \quad(2.4)$ & \multirow{3}{*}{0.68} \\
\hline AT & $34(16.0)$ & $55(25.9)$ & $22(10.4)$ & \\
\hline $\mathrm{AA}$ & $15 \quad(7.1)$ & $35(16.5)$ & $12(5.7)$ & \\
\hline \multicolumn{5}{|l|}{ NOD2 } \\
\hline rs 18617 & & & & \multirow{4}{*}{0.22} \\
\hline TT & $17 \quad(8.4)$ & $38(18.7)$ & 14 (6.9) & \\
\hline TG & $34(16.7)$ & $41(20.2)$ & $21(10.3)$ & \\
\hline GG & $9(4.4)$ & $24(11.8)$ & $5(2.5)$ & \\
\hline G & $52 \quad(2.8)$ & 89 (21.6) & $31 \quad(7.6)$ & 070 \\
\hline $\mathrm{T}$ & $68(16.7)$ & $117(28.8)$ & $49(12.1)$ & $0 . / 8$ \\
\hline$r s 31355$ & & & & \\
\hline GG & $11 \quad(6.7)$ & $25(15.3)$ & $12(7.4)$ & \\
\hline GA & $25 \quad 15.3)$ & $39(23.9)$ & 14 (8.6) & 0.79 \\
\hline $\mathrm{AA}$ & $10 \quad(6.1)$ & $20(12.3)$ & $7 \quad(4.3)$ & \\
\hline$I L-1 \beta$ & & & & \\
\hline$r s 16944$ & & & & \\
\hline GG & $24(12.3)$ & $44(22.6)$ & $14(7.2)$ & \\
\hline GA & $21(10.8)$ & 48 (24.6) & $19 \quad(9.7)$ & $0 . / 6$ \\
\hline $\mathrm{AA}$ & $9(4.6)$ & $12(6.2)$ & $4(2.1)$ & \\
\hline$r s 11436$ & & & & \\
\hline GG & $8 \quad(4.0)$ & $13 \quad(6.5)$ & $4 \quad(2.0)$ & \\
\hline GA & $27(13.5)$ & $50(25.0)$ & $21(10.5)$ & 0.94 \\
\hline $\mathrm{AA}$ & $22(11.0)$ & $42(21.0)$ & $13(6.5)$ & \\
\hline rs 10865 & & & & \\
\hline GG & $2 \quad(0.9)$ & $2 \quad(0.9)$ & $0 \quad(0.0)$ & \\
\hline GC & $23(10.8)$ & $30(14.2)$ & 10 & 0.33 \\
\hline $\mathrm{CC}$ & $36(17.0)$ & $77(36.3)$ & $32(15.1)$ & \\
\hline $\begin{array}{l}\text { PPARY } \\
\quad r s 18012\end{array}$ & & & & \\
\hline GC & $6 \quad(2.7)$ & $6 \quad(2.7)$ & $1 \quad(0.5)$ & \\
\hline $\mathrm{CC}$ & $60(27.4)$ & $105(47.9)$ & 41 (18.7) & 0.32 \\
\hline $\mathrm{CC}$ & $58(26.0)$ & $107(48.0)$ & $41(18.4)$ & \\
\hline$r s 38568$ & & & & \\
\hline CC & $58(26.0)$ & $107(48.0)$ & $41(18.4)$ & \\
\hline CT & $9 \quad(4.0)$ & $7 \quad(3.1)$ & $1(0.4)$ & 0.08 \\
\hline TT & $\begin{array}{ll}0 & (0.0)\end{array}$ & $\begin{array}{ll}0 & (0.0)\end{array}$ & $\begin{array}{ll}0 & (0.0)\end{array}$ & \\
\hline
\end{tabular}

A previous meta-analysis in Asian patients reported that omeprazole-based therapies were influenced by the different genotypes of the CYP2C19, while lansoprazolebased treatments were not. ${ }^{11,23,24}$ Nevertheless, this analysis revealed that the studied SNPs of the CYP2C19 gene in the Jordanian Arab descent population did not show any significant correlation with the type of drugs used and the responsiveness to the treatment.

The genotypes of IL-1 $\beta$ rs 16944 potentiate the synthesis of cytokines and are significantly correlated with the clinical development of $H$. pylori infection. ${ }^{9,25,26}$ The presence of the A allele of the IL-1 $\beta$ rs1143627 showing a higher chance of developing the genotype AG in a German population. ${ }^{27}$ The same result was obtained from the Caucasian and Brazilian populations that IL-1 $\beta$ (AA) and (AG) genotypes of rs1143627 SNP variant were correlated with chronic gastritis and gastric tumor progression in individuals infected with $H$. pylori, $8,27,28$ but the current research showed no association between the genotypes IL-1 $\beta$ rs 16944 and rs1143627 and the distribution between healthy individuals and peptic ulcer patients, in addition to the severity of the $H$. pylori infection. These findings are consistent to other research on the European and Mexican populations. ${ }^{29}$

Additionally, several researchers have found that modifications in the TNF- $\alpha$ gene promoter region are correlated with a greater risk of promoting gastritis. ${ }^{9,20}$ The TNF- $\alpha$ produced in the gastric submucosa by macrophages plays a major role in the regulation of gastric acid secretion, which is one of the main factors of duodenal diseases development due to infection with H. pylori. ${ }^{30,31}$ The environment of low acid secretion and aggressive inflammatory response can enhance the $H$. pylori colonization in the gastric mucosa. ${ }^{9}$ Results of the meta-analysis specify that the TNF- $\alpha-308$ $(\mathrm{G}>\mathrm{A}) \quad(\mathrm{rs} 1800629)$ and $-1031 \quad(\mathrm{~T}>\mathrm{C})$ (rs 1799964) polymorphisms may be associated with a reduced risk of $H$. pylori infection in the Chinese population. ${ }^{29} \mathrm{It}$ has been found in the Japanese population that the $\mathrm{C} / \mathrm{C}$ and T/C genotypes of the TNF- $\alpha$ rs 1800629 were at the greatest risk from $H$. pylori infections. ${ }^{31,32}$ Our results about the TNF- $\alpha$ polymorphisms (rs1799964, rs1800629, and rs361525) matched the results from other studies in Asian, European, and Caucasian populations. ${ }^{9,29}$ In this study, the results show no statistical evidence of a considerable correlation between the vulnerability to $H$. pylori infections in gastric ulcer patients, activity, and severity in the Jordanian Arab population.

The NOD1 and NOD2 genes might play a crucial role in $H$. pylori-related processes of inflammation and 
carcinogenesis. ${ }^{33}$ Therefore, variations in these 2 genes could affect chronic inflammation caused by $H$. pylori and increase the risk of gastric cancer. ${ }^{7}$ It was found that rs2907749 and rs3135500 were associated with the risk of gastric ulcers. ${ }^{33}$ Many studies suggest the (TT) homozygote genotype of the polymorphism (rs2075820) found in the NOD1 gene raises the potential risk of development of peptic ulceration in the patients diagnosed with $H$. pylori-positive in the Hungarian population. ${ }^{7,34}$ Many studies also suggest that if the structure or regulation of the NOD1 protein is altered, this would change the reactivity to $H$. pylori and the nature of downstream inflammatory mechanisms. ${ }^{7}$ This study has investigated the relationship of 3 mutations in NOD1 (rs2075820, rs2907749, and rs7789045) gene and 2 mutations (rs1861759 and rs3135500) in NOD2 gene with the risk to $H$. pylori infections in the Jordanian Arab population. This study revealed that the individuals who carry the heterozygous genotype (TC) rs2075820 have a decreased risk of developing peptic ulcers (odd ratio: $0.49, p=0.0046$ ). The allele frequency in the patients were $66 \%$ (C) and $34 \%$ (T), compared to $60 \%(\mathrm{C})$ and $40 \%(\mathrm{~T})$ in healthy individuals with insignificant association. Many studies have shown that having the (A) allele at rs3135500 was significantly connected with a slightly decreased chance of developing a gastric ulcer in the Chinese population, but the NOD2 mutations did not show any significant association either in genotype or allele models with $H$. pylori infection. ${ }^{6,9}$ The variation of our results from other reported results could be due to the sample size and the source type of DNA and ethnicity. A small number of studies were conducted in this field concerning $H$. pylori infection in the Arab population. It is approved that the FFPE tissue is an important source of genetic material that can be used for molecular and pharmacogenetic studies. Therefore, the FFPE tissue could represent an alternative source of DNA instead of peripheral blood samples as for retrospective studies. The genetic material quality and quantity that extracted from FFPE is low compared with that extracted from fresh tissues, but this problem could be overcome by the optimization of the methods that used for DNA extraction and amplification. Furthermore, this type of studies is limited by the lack of clinical information for the studied patients.

In conclusion, this study analyzed the genetic association of many genetic variants with the risk of H. pylori infection and the response to the treatments in the Jordanian Arab population. TLR-10 rs10004195 SNP was associated with decreasing the risk of $H$. pylori infections; others, such as NOD1 rs2075820 SNP,
TLR-4 rs10759932 SNP, and TNF- $\alpha$ rs361525 and rs 1799964 SNPs showed a significant association with the responsiveness to the type of treatment prescribed to the patients by the consultant. However, a high percentage of the types of medications written to the patients did not depend on the response of the patients to the prescribed drugs. Internationally, a phenomenon of $H$. pylori isolates resisting the antibiotics has been proved and should be considered to develop an optimal therapy. Therefore, these types of genetic association analyses can be used for matching patients into different treatment options based on their genetic profiles to improve medical treatment strategy used for treating ulcer patients.

Acknowledgment. This study was funded by Jordan University of Science and Technology (R\#: 148/2017).

\section{References}

1. Stolte M, Meining A. The updated Sydney system: Classification and grading of gastritis as the basis of diagnosis and treatment. Can J Gastroenterol 2001; 15: 591-598.

2. Graham DY. Helicobacter pylori update: Gastric cancer, reliable therapy, and possible benefits. Gastroenterology 2015; 148: 719-731.

3. Khan AR. Comparison of $\mathrm{H}$. pylori-gastritis among young and old patients by using" the modified Sydney system of classification and grading". Saudi J Gastroenterol 1999; 5: 81-84.

4. Mitchell H, Katelaris P. Epidemiology, clinical impacts and current clinical management of Helicobacter pylori infection. Med J Aust 2016; 204: 367-380.

5. Goudarzi H, Seyedjavadi SS, Fazeli M, Azad M, Goudarzi M. Genotyping of peroxisome proliferator-activated receptor gamma in iranian patients with Helicobacter pylori infection. Asian Pacific J Cancer Prev 2015; 16: 5219-5223.

6. Ram MR, Goh KL, Leow AH, Poh BH, Loke MF, Harrison $\mathrm{R}$, et al. Polymorphisms at locus $4 \mathrm{p} 14$ of Toll-like receptors TLR-1 and TLR-10 confer susceptibility to gastric carcinoma in Helicobacter pylori infection. PLoS One 2015; 10: 1-15.

7. Wang P, Zhang L, Jiang JM, Ma D, Tao HX, Yuan SL, et al. Association of NOD1 and NOD2 genes polymorphisms with Helicobacter pylori related gastric cancer in a Chinese population. World J Gastroenterol 2012; 18: 2112-2120.

8. Drici AE, Moulessehoul S, Tifrit A, Diaf M, Turki DK, Bachir $M$, et al. Effect of IL-1 $\beta$ and IL-1RN polymorphisms in carcinogenesis of the gastric mucosa in patients infected with Helicobacter pylori in Algeria. Libyan J Med 2016; 11: 1-7.

9. Santos JC, Ladeira MS, Pedrazzoli J, Ribeiro ML. Relationship of IL- 1 and TNF- $\alpha$ polymorphisms with Helicobacter pylori in gastric diseases in a Brazilian population. Brazilian J Med Biol Res 2012; 45: 811-817.

10. Han R, Lu H, Jiang MW, Tan KW, Peng Z, Hu JL, et al. Multicenter Study of Antibiotic Resistance Profile of $H$. pylori and Distribution of CYP2C19 Gene Polymorphism in Rural Population of Chongqing, China. Gastroenterol Res Pract 2016; 2016: 1-6. 
11. Kuo CH, Lu CY, Shih HY, Liu CJ, Wu MC, Hu HM, et al. CYP2C19 polymorphism influences Helicobacter pylori eradication. World J Gastroenterol 2014; 20: 16029-16036.

12. Hunfeld NG, Touw DJ, Mathot RA, Van Schaik RH, Kuipers EJ. A comparison of the acid-inhibitory effects of esomeprazole and rabeprazole in relation to pharmacokinetics and CYP2C19 polymorphism. Aliment Pharmacol Ther 2012; 35: 810-818.

13. Jainan W, Vilaichone RK. Effects of the CYP2C19 genetic polymorphism on gastritis, peptic ulcer disease, peptic ulcer bleeding and gastric cancer. Asian Pacific J Cancer Prev 2014; 15: 10957-10960.

14. Saxena A, Shukla SK, Prasad KN, Ghoshal UC. Analysis of p53, K-ras gene mutation \& Helicobacter pylori infection in patients with gastric cancer \& peptic ulcer disease at a tertiary care hospital in north India. Indian J Med Res 2012; 136: 664-670.

15. Belda S, Saez J, Santibáñez M, Rodríguez JC, Sola-Vera J, Ruiz-García M, et al. Relationship between bacterial load, morbidity and cagA gene in patients infected by Helicobacter pylori. Clin Microbiol Infect 2012; 18: E251-E253.

16. Biernat MM, Gosciniak G, Iwanczak B. Prevalence of Helicobacter pylori cagA, vacA, iceA, babA2 genotypes in Polish children and adolescents with gastroduodenal disease. Advances in Hygiene \& Experimental Medicinel PostepyHigienyiMedycynyDoswiadczalnej 2014; 68: 1015-1021.

17. Suzuki H, Mori H. World trends for $H$. pylori eradication therapy and gastric cancer prevention strategy by $H$. pylori testand-treat. J Gastroenterol 2018; 53: 354-361.

18. Bridge DR, Merrell DS. Polymorphism in the Helicobacter pylori CagA and VacA toxins and disease. Gut microbes 2013; 4: 101-117.

19. Fashner J, Gitu AC. Diagnosis and treatment of peptic ulcer disease and H. pylori infection. Am Fam Physician 2015; 91: 236-242.

20. Dunne C, Dolan B, Clyne M. Factors that mediate colonization of the human stomach by Helicobacter pylori. World J Gastroenterol 2014; 20: 5610-5624.

21. Sychev DA, Denisenko NP, Sizova ZM, Grachev AV, Velikolug KA. The frequency of CYP2C19 genetic polymorphisms in Russian patients with peptic ulcers treated with proton pump inhibitors. Pharmgenomics Pers Med 2015; 8: 111-114.

22. Lin YA, Wang H, Gu ZJ, Wang WJ, Zeng XY, Du YL, et al. Effect of CYP2C19 gene polymorphisms on proton pump inhibitor, amoxicillin, and levofloxacin triple therapy for eradication of Helicobacter Pylori. Med Sci Monit 2017; 23: 2701-2707.

23. Kuo CH, Liu CJ, Yang CC, Kuo FC, Hu HM, Shih HY, et al. A rapid and accurate method to evaluate Helicobacter pylori infection, clarithromycin resistance, and CYP2C19 genotypes simultaneously from gastric juice. Med (United States) 2016; 95: $1-7$.
24. Velin D, Straubinger K, Gerhard M. Inflammation, immunity, and vaccines for Helicobacter pylori infection. Helicobacter 2016; 21: 26-29.

25. Sonnenberg A, Turner KO, Spechler SJ, Genta RM. The influence of Helicobacter pylori on the ethnic distribution of Barrett's metaplasia. Aliment Pharmacol Ther 2017; 45: 283-290.

26. Varga MG, Piazuelo MB, Romero-Gallo J, Delgado AG, Suarez G, Whitaker ME, et al. TLR9 activation suppresses inflammation in response to Helicobacter pylori infection. Am J Physiol - Gastrointest Liver Physiol 2016; 311: G852-G858.

27. Li X, Liu S, Luo J, Liu A, Tang S, Liu S, et al. Helicobacter pylori induces IL- $1 \beta$ and IL-18 production in human monocytic cell line through activation of NLRP3 inflammasome via ROS signaling pathway. Pathog Dis 2015; 73: 1-8.

28. Kameoka S, Kameyama T, Hayashi T, Sato S, Ohnishi N, Hayashi $\mathrm{T}$, et al. Helicobacter pylori induces IL-1 $\beta$ protein through the inflammasome activation in differentiated macrophagic cells. Biomed Res 2016; 37: 21-27.

29. Murphy G, Thornton J, McManus R, Swan N, Ryan B, Hughes DJ, O'Morain CA, et al. Association of gastric disease with polymorphisms in the inflammatory related genes IL-1B, IL-1RN, IL-10, TNF and TLR4. Eur J Gastroenterol Hepatol. 2009; 21: 630-635.

30. Chen G, Tang N, Wang C, Xiao L, Yu M, Zhao L, et al. TNF$\alpha$-inducing protein of Helicobacter pylori induces epithelialmesenchymal transition (EMT) in gastric cancer cells through activation of IL-6/STAT3 signaling pathway. Biochem Biophys Res Commun 2017; 484: 311-317.

31. Siregar GA, Halim S, Sitepu RR. Serum TNF-a, IL-8, VEGF levels in Helicobacter pylori infection and their association with degree of gastritis. Acta Med Indones 2015; 47: 120-126.

32. You W, Lai X, Lv J, Chen P, Xia J, Cui F, et al. Association of tumor necrosis factor- $\alpha$ gene polymorphisms with susceptibility to Helicobacter pylori-associated gastroduodenal diseases in the Chinese population. Int J Clin Exp Pathol 2016; 9: 12836-12842.

33. Li ZX, Wang YM, Tang FB, Zhang L, Zhang Y, Ma JL, et al. NOD1 and NOD2 genetic variants in association with risk of gastric cancer and its precursors in a Chinese population. PLoS One 2015; 10: 1-14.

34. Tran LS, Tran D, De Paoli A, D’Costa K, Creed SJ, Ng GZ, et al. NOD1 is required for Helicobacter pylori induction of IL-33 responses in gastric epithelial cells. Cell Microbiol 2018; 20: $1-12$. 